\title{
Impactos Concorrenciais da Entrada dos Medicamentos Genéricos no Mercado Farmacêutico Brasileiro de 2003 a 2007
}

\section{Competitive Impacts after Generic Medicines Entry in the Brazilian Pharmaceutical Market between 2003 and 2007}

\author{
Alexis Toríbio Dantas* \\ Pedro Alerrandro Saccol Mendonça**
}

\begin{abstract}
Resumo: Em 1999, o mercado de medicamentos genéricos foi criado no Brasil, iniciando-se, assim, uma nova fase do mercado farmacêutico brasileiro. Através de dados cedidos pela Anvisa sobre o mercado varejista brasileiro, objetiva-se testar até que ponto a política de medicamentos genéricos tem logrado êxito. Foram utilizados dados em painel para analisar o impacto da entrada dos medicamentos genéricos sobre a estrutura do mercado, assim como o impacto nos preços e na quantidade vendida entre 2003 e 2007. Os resultados foram favoráveis à diminuição da concentração dos mercados relevantes da amostragem e também ao aumento da quantidade comercializada. Em relação aos preços, o modelo se mostrou pouco significativo, provavelmente pela política de medicamentos genéricos ser um instrumento indireto de controle de preços.
\end{abstract}

Palavras-chave: Regulação. Mercado farmacêutico. Medicamentos genéricos. Mercado relevante.

Abstract: In 1999, the generic medicines market was created in Brazil. Thus, a new phase of the Brazilian pharmaceutical market began. Through data provided by Anvisa on the Brazilian retail market, aimed to test in how far the generic drug policy has been successful, we used panel data to analyze the impact of the entry of generic drugs on the market structure, as well as the impact on prices and quantities sold between 2003 and 2007. The results were favorable to the policy as they show decrease of the concentration level in the relevant markets of the sample and also increase of the quantity sold. Regarding prices, the model was negligible, probably due to generic drug policy being an instrument of indirect price control.

Keywords: Regulation. Pharmaceutical market. Generic drugs. Relevant market.

JEL Classification: L10.

* $\quad$ Doutor em Economia pela Universidade Federal do Rio de Janeiro (UFRJ). Professor associado da Faculdade de Ciências Econômicas da Universidade do Estado do Rio de Janeiro (UERJ). E-mail: alexis.dantas@gmail.com

* $\quad$ Mestre em Ciências Econômicas pela Universidade do Estado do Rio de Janeiro (UERJ). E-mail: pedroasm@superig.com.br 


\section{Introdução}

Consideradas as características essenciais da indústria farmacêutica, sempre houve grande importância em regular os preços do setor, visando garantir o acesso da população como um todo a um bem tão fundamental. Uma forma de regulação dos preços tem sido a introdução do mercado de produtos genéricos, medicamentos cujas patentes já expiraram, sendo vendidos sob a denominação genérica do princípio ativo, visando o aumento da concorrência e, consequentemente, a diminuição dos preços, portanto, atuando como uma forma de regulação indireta sobre os preços.

No Brasil, desde 1994, o esforço de se regulamentar o medicamento genérico tem existido, porém somente em 1999 que a regulação de fato ocorreu, com a Lei 9.787/99, introduzindo o mercado de medicamentos genéricos. Avaliações sobre esse mercado sinalizam um aparente sucesso da política, sendo válido analisar na literatura existente a possibilidade da atualização do debate.

O objetivo deste trabalho é inferir se a entrada desses medicamentos influenciou um aumento da concorrência no setor, possibilitando a redução dos preços. Ao mesmo tempo, verificar se a inserção aumentou o volume de vendas de forma a aumentar o bem-estar social, uma vez que, se a transformação se desse apenas ao nível dos preços, estaria ocorrendo uma simples troca de excedente entre consumidor e produtor.

Este artigo se divide em quatro seções: a primeira seção, esta introdução, apresenta o tema a ser dissertado; a segunda seção analisa os impactos concorrenciais relacionados aos medicamentos genéricos na indústria farmacêutica, ${ }^{1}$ sendo apresentadas as características de oferta e de demanda, a estrutura do mercado, as estratégias tomadas pelas empresas, o desempenho e o que se espera que o poder público assuma como suas responsabilidades; a terceira seção avalia as expectativas de regulação do mercado de medicamentos, tendo os genéricos como instrumento no Brasil, além de abordar a metodologia, as hipóteses fundamentais e a fonte dos dados utilizados e apresentar os testes e os resultados dos impactos concorrenciais da entrada dos medicamentos genéricos sobre a estrutura de mercado, os preços dos medicamentos e a variação da quantidade comercializada separadamente; para finalizar, a quarta seção apresenta as considerações finais.

1 O referencial teórico para a análise dos principais pontos sobre a indústria farmacêutica associa-se ao modelo estrutura-conduta-desempenho desenvolvido por Bain (1959) e difundido por Scherer (1970) e seus desdobramentos. Ver também Davies et al. (1988). 


\section{Impactos Concorrenciais da Entrada dos Medicamentos Genéricos no Mercado Farmacêutico Brasileiro}

\subsection{A Experiência Brasileira com a Regulação de Genéricos}

Em 1999, o governo federal promulgou a Lei 9.782/99, que define o Sistema Nacional de Vigilância Sanitária e que levou à criação da Agência Nacional de Vigilância Sanitária (Anvisa), cuja função era a implementação e a execução da política nacional de vigilância sanitária, sendo atribuída posteriormente, por medida provisória, também a função sobre as questões relacionadas aos preços dos medicamentos.

As principais alterações realizadas pela Lei 9.787/99 (UNIVERSIDADE FEDERAL DO RIO DE JANEIRO, 2002) foram:

a) instituir a exigência de que todos os medicamentos comercializados com marca também ostentem, nas embalagens e materiais promocionais, a denominação do fármaco ou princípio farmacologicamente ativo com uma dimensão não inferior à metade do tamanho das letras e caracteres da marca;

b) atribuir ao Ministério da Saúde a competência para promover a difusão de informações sobre o uso de medicamentos genéricos;

c) obrigar que as aquisições de medicamentos e as prescrições médicas/odontológicas de medicamentos, no âmbito do Sistema Único de Saúde (SUS), adotem a denominação do princípio ativo;

d) obrigar o órgão federal responsável pela vigilância sanitária a editar, periodicamente, a relação de medicamentos registrados no país, de acordo com a classificação farmacológica da Relação Nacional de Medicamentos Essenciais (Rename) vigente;

e) estabelecer preferência ao medicamento genérico nas compras do SUS quando houver igualdade de preço e demais condições de aquisição;

f) estabelecer que os medicamentos similares devem ser sempre identificados por nome comercial ou marca;

g) prever regulamentação especial sobre os medicamentos genéricos pelo órgão federal de vigilância sanitária. 
Até a edição da Lei 9.787/99, a regulamentação do registro de medicamentos genéricos inexistia. Existiam, entretanto, os medicamentos similares aos medicamentos de marca que eram vendidos pelo princípio ativo, mas sem a garantia técnica de bioequivalência. Somente depois de formada uma base preocupada com a qualidade e o bom uso dos medicamentos foi criada a Lei dos Genéricos. ${ }^{2}$

Através da regulamentação da Lei dos Genéricos, foi aprovado o Regulamento Técnico para Medicamentos Genéricos, que estabelece os critérios e as condições para o registro e o controle de qualidade de genéricos, para os testes de biodisponibilidade e bioequivalência e para a dispensação de medicamentos genéricos nos serviços farmacêuticos públicos e privados. Ao mesmo tempo, visou-se controlar os preços dos medicamentos por meio do aumento da competição no mercado, ou seja, uma forma de controle indireto sobre os preços.

Em dezembro de 2000, com a edição da Medida Provisória no 2.063, o governo retomou o controle dos preços do setor farmacêutico, partindo para uma forma de controle direto. Na ocasião, foram definidas as normas de regulação para os preços dos medicamentos e a Câmara de Medicamentos (Camed) foi criada, sendo um órgão interministerial composto de ministros da Casa Civil, Justiça, Fazenda e Saúde e um comitê técnico envolvendo secretários de gestão de investimentos em saúde (MS), direito econômico (SDE/MJ), acompanhamento econômico (Seae/MF) e um representante da Casa Civil.

A atuação da Camed durou até junho de 2003, quando foi substituída pela Câmara de Regulação do Mercado de Medicamentos (CMED), criada por meio do Decreto $n^{\circ} 4.766$, de 26 de junho de 2003, com a função de regular o mercado e estabelecer os critérios para a definição e o ajuste de preços de medicamentos.

Considerando essas medidas legais como o arcabouço do mercado de medicamentos genéricos brasileiro e após relevar os três exemplos de política de regulação, parte-se para o teste empírico que tentará analisar até que ponto a regulação brasileira está atingindo aquilo que se espera, ou seja, aumentar o bem-estar da população.

\subsection{Bases do modelo}

Um dos maiores desafios da análise econômica na indústria farmacêutica está na definição do mercado para inferir o poder de monopólio das empresas. Em geral, esse poder se mostra pouco importante quando calculado para o mercado farmacêutico como um todo. Por outro lado, quando o mercado é redefinido por princípios ativos semelhantes ou classificações terapêuticas, a verdadeira participação das vendas de cada empresa desse mercado segmentado se mostra bastante elevado.

$2 \quad$ Como ficou sendo conhecida a Lei 9.787/99, de 11 de fevereiro de 1999. 
Para superar o obstáculo da definição do mercado é comum utilizar o conceito de mercado relevante. Esse mercado inclui somente os produtos e as empresas que concorrem entre si, ou seja, inclui somente os substitutos entre si. Sobre a ótica da demanda, os concorrentes entre si são os destinados aos mesmos usos e, sobre a ótica da oferta, são aqueles medicamentos produzidos com tecnologias ou matérias-primas semelhantes.

Seguindo esse princípio, o segmento de mercado em que realmente há concorrência entre os medicamentos pode ser explicitado através da classe terapêutica do medicamento como fronteira de concorrência. No entanto, ainda podem ser encontrados medicamentos com pouco ou nenhum grau de substituibilidade nesse nível de agregação. Uma alternativa seria descer ao nível de segmentação do mercado por princípio ativo, ou matéria-prima do medicamento, porém haveria o problema de a classificação ficar muito estreita, havendo princípios ativos diferentes que seriam substitutos muito próximos entre si, e, ainda, princípios ativos iguais, mas com uma forma farmacêutica diferente, que não se apresentariam como substitutos.

A escolha da abrangência do mercado relevante envolve um amplo conhecimento dos usos dos medicamentos e de suas características tecnológicas. Calibrar esses dois elementos na definição do mercado relevante é uma boa defesa contra a incorrência de erro de avaliação no grau de monopólio praticado pelas empresas em cada mercado. Uma definição muito estreita do mercado relevante levaria a uma superestimação desse poder e, em uma situação inversa, a uma definição muito ampla, a uma subestimação.

\subsection{Metodologia}

Para a determinação dos mercados relevantes, utilizou-se a base de dados fornecida pela Anvisa como fonte das informações sobre o mercado varejista de medicamentos para o período entre 2003 e 2007, discriminados mês a mês. A listagem de produtos seguia classificação própria baseada na classificação ATC (Anatomical, Therapeutic, Chemical) da WHO (World Health Organization), voltada para um completo inventário dos produtos, ao invés de indicar o grau de substituição entre os mesmos.

A partir da classificação ATC, optou-se por identificar as correspondências dessa classificação com a Rename, ${ }^{3}$ pois esta define a organização por grupos farmacológicos a partir do uso clínico dos medicamentos, o que facilita o enquadramento dos medicamentos na definição dos mercados relevantes.

3 Contou-se com a colaboração de André Reis, farmacêutico, Beatriz Fialho, economista, ambos do Grupo de Economia da Inovação, e Aroldo Mendonça, médico, para este procedimento. 
Especificamente para o caso de produtos com mais de um princípio ativo, ou seja, associações medicamentosas, procurou-se estabelecer o uso terapêutico da associação em questão. Quando o consenso não era obtido, classificou-se a associação pelo principal princípio ativo da formulação.

Uma das preocupações na análise dos impactos concorrenciais na indústria farmacêutica era identificar os mercados relevantes mais críticos em termos de importância para a população. Para isso, foram observados os indicadores e dados básicos do Ministério da Saúde (2008) sobre as principais causas de mortalidade e morbidade no Brasil, representados nas Tabelas 1 e 2.

Tabela 1 - Prevalência de óbitos no Brasil em 2006

\begin{tabular}{lcc}
\hline \multicolumn{1}{c}{ Grupos de doenças } & Número de óbitos & Proporção de óbitos \\
\hline $\begin{array}{l}\text { Doenças do aparelho } \\
\text { circulatório }\end{array}$ & 302.817 & $32,01 \%$ \\
$\begin{array}{l}\text { Neoplasias (tumores) } \\
\text { Causas externas }\end{array}$ & 155.796 & $16,47 \%$ \\
$\begin{array}{l}\text { Doenças do aparelho } \\
\text { respiratório }\end{array}$ & 128.388 & $13,57 \%$ \\
$\begin{array}{l}\text { Doenças infecciosas e } \\
\text { parasitárias }\end{array}$ & 102.866 & $10,87 \%$ \\
$\begin{array}{l}\text { Afecções originadas no } \\
\text { período perinatal }\end{array}$ & 46.508 & $4,92 \%$ \\
$\begin{array}{l}\text { Demais doenças defini- } \\
\text { das }\end{array}$ & 181.437 & $2,99 \%$ \\
\hline Total & 946.148 & $19,18 \%$ \\
\hline
\end{tabular}

Fonte: Brasil (2009).

Tabela 2 - Prevalência de internações no Brasil em 2006

\begin{tabular}{lcc}
\hline \multicolumn{1}{c}{ Grupos de doenças } & $\begin{array}{c}\text { Número de } \\
\text { internações }\end{array}$ & $\begin{array}{c}\text { Proporção de } \\
\text { internações }\end{array}$ \\
\hline $\begin{array}{l}\text { Doenças durante a } \\
\text { gravidez, parto ou } \\
\text { puerpério }\end{array}$ & 2.527 .056 & $22,29 \%$ \\
$\begin{array}{l}\text { Doenças do aparelho } \\
\text { respiratório }\end{array}$ & 1.541 .113 & $13,59 \%$ \\
$\begin{array}{l}\text { Doenças do aparelho } \\
\text { circulatório }\end{array}$ & 1.146 .867 & $10,12 \%$ \\
\hline & & continua...
\end{tabular}


conclusão..

\begin{tabular}{lcc}
\hline \multicolumn{1}{c}{ Grupos de doenças } & $\begin{array}{c}\text { Número de } \\
\text { internações }\end{array}$ & $\begin{array}{c}\text { Proporção de } \\
\text { internações }\end{array}$ \\
\hline $\begin{array}{l}\text { Doenças infecciosas e } \\
\text { parasitárias }\end{array}$ & 996.549 & $8,79 \%$ \\
$\begin{array}{l}\text { Doenças do aparelho } \\
\text { digestivo }\end{array}$ & 979.474 & $8,64 \%$ \\
Causas externas & 793.823 & $7,00 \%$ \\
$\begin{array}{l}\text { Doenças do aparelho } \\
\text { geniturinário }\end{array}$ & 745.185 & $6,57 \%$ \\
$\begin{array}{l}\text { Neoplasias (tumores) } \\
\text { Transtornos mentais e } \\
\text { comportamentais }\end{array}$ & 614.003 & $5,42 \%$ \\
Demais causas & 317.441 & $2,80 \%$ \\
\hline Total & 1.676 .526 & $14,79 \%$ \\
\hline
\end{tabular}

Fonte: Brasil (2009b).

Segundo a lista de medicamentos cujos preços estão em conformidade com a legislação da CMED, o mercado farmacêutico brasileiro é composto por 284 laboratórios e 19.531 produtos. Conforme a Tabela 3, as vendas, em valores nominais, evoluíram de $\mathrm{R} \$ 14,8$ bilhões, em 2003, para $\mathrm{R} \$ 23,6$ bilhões, em 2007, e, em quantidade, de 1,2 bilhão de unidades para 1,5 bilhão.

Tabela 3 - Evolução das vendas de medicamentos no Brasil entre 2003 e 2007

\begin{tabular}{ccccc}
\hline Ano & $\begin{array}{c}\text { Vendas } \\
\text { (em \$1.000) }\end{array}$ & $\begin{array}{c}\text { Variação } \\
\mathbf{( \% )}\end{array}$ & $\begin{array}{c}\text { Vendas } \\
\text { (em 1.000 unidades) }\end{array}$ & $\begin{array}{c}\text { Variação } \\
\text { (\%) }\end{array}$ \\
\hline 2003 & 4.780 .035 & & 1.219 .059 & \\
2004 & 17.254 .160 & 16,7 & 1.332 .963 & 9,3 \\
2005 & 19.232 .249 & 11,5 & 1.374 .337 & 3,1 \\
2006 & 21.453 .166 & 11,5 & 1.436 .958 & 4,6 \\
2007 & 23.581 .666 & 9,9 & 1.517 .370 & 5,6 \\
\hline
\end{tabular}

Fonte: Abimip (2009).

Após o levantamento da primeira triagem, o conjunto de observações totalizou 6.536 produtos, produzidos por 180 laboratórios, correspondendo a um faturamento nominal de $\mathrm{R} \$ 3,2$ bilhões, em 2003, e R $\$ 4,5$ bilhões, em 2007, nos segmentos ético e não ético. Em termos de quantidade de medicamentos vendi- 
dos, a amostragem apontou para a comercialização de 304,4 milhões de unidades, em 2003, e de 361,9 milhões, em 2007, como mostra a Tabela 4.

Tabela 4 - Evolução das vendas de medicamentos da amostra de mercado analisado entre 2003 e 2007

\begin{tabular}{ccccc}
\hline Ano & $\begin{array}{c}\text { Vendas } \\
\text { (em \$1.000) }\end{array}$ & Variação (\%) & $\begin{array}{c}\text { Vendas } \\
\text { (em 1.000 } \\
\text { unidades) }\end{array}$ & Variação (\%) \\
\hline 2003 & 3.194 .593 & & 304.429 & \\
2004 & 3.606 .698 & 12,9 & 325.230 & 6,8 \\
2005 & 3.913 .961 & 8,5 & 337.126 & 3,7 \\
2006 & 4.200 .253 & 7,3 & 350.171 & 3,9 \\
2007 & 4.462 .214 & 6,2 & 361.855 & 3,3 \\
\hline
\end{tabular}

Fonte: Abimip (2009).

Em função dos resultados obtidos, deduz-se que a amostra analisada corresponde a cerca de $20 \%$ do mercado total em valor e a $24 \%$ do mercado total em quantidade. Essa comparação pode ser melhor observada com o auxílio da Tabela 5.

Tabela 5 - Representatividade da amostra analisada no mercado farmacêutico brasileiro total

\begin{tabular}{ccccccc}
\hline Ano & $\begin{array}{c}\text { Vendas } \\
\text { Totais }\end{array}$ & $\begin{array}{c}\text { Vendas } \\
\text { da } \\
\text { Amostra }\end{array}$ & $\begin{array}{c}\text { Partici- } \\
\text { pação }\end{array}$ & $\begin{array}{c}\text { Vendas } \\
\text { Totais }\end{array}$ & $\begin{array}{c}\text { Vendas } \\
\text { da } \\
\text { Amostra }\end{array}$ & $\begin{array}{c}\text { Partici- } \\
\text { pação }\end{array}$ \\
\hline & \multicolumn{2}{c}{ (em R\$1.000) } & (\%) & \multicolumn{2}{c}{ (em R\$1.000) } & (\%) \\
\hline 2003 & 14.780 .035 & 3.194 .593 & 21,6 & 1.219 .059 & 304.429 & 25 \\
2004 & 17.254 .160 & 3.606 .698 & 20,9 & 1.332 .963 & 325.230 & 24,4 \\
2005 & 19.232 .249 & 3.913 .961 & 20,4 & 1.374 .337 & 337.126 & 24,5 \\
2006 & 21.453 .166 & 4.200 .253 & 19,6 & 1.436 .958 & 350.171 & 24,4 \\
2007 & 23.581 .666 & 4.462 .214 & 18,9 & 1.517 .370 & 361.855 & 23,8 \\
\hline
\end{tabular}

Fonte: Elaboração própria a partir de dados da Anvisa.

Ao fim dessa primeira etapa, 94 princípios ativos foram distribuídos entre 45 mercados relevantes. Sempre que se entendeu como necessário, novas subdivisões da Rename foram criadas, porém diferenças nas apresentações, como a 
forma farmacêutica e a concentração do princípio ativo, não foram levadas em consideração, sendo uma importante limitação deste trabalho. ${ }^{4}$

Uma segunda etapa consistiu na contagem do número de produtos genéricos, de medicamentos de referência, de princípios ativos e laboratórios dentro de cada mercado relevante, assim como foi calculado o grau de concentração do mesmo através do índice de Herfindahl-Hirschman (HHI). ${ }^{5}$

Conforme as diretrizes para a política antitruste norte-americana (UNITED STATES OF AMERICA, 2009), mercados com HHI inferior a 1.000 são considerados competitivos, aqueles com HHI entre 1.000 e 1.800 são considerados com concentração moderada e os superiores a 1.800 são entendidos como altamente concentrados. Um monopólio, por definição, possui HHI igual a 10.000 .

\subsubsection{As Hipóteses}

A política de introdução de medicamentos genéricos no mercado farmacêutico visa combinar três efeitos como em uma reação em cadeia. O primeiro efeito seria o aumento do grau de concorrência nos mercados relevantes em que se dá a entrada de novos produtos. Alcançada essa primeira etapa, a redução do poder de monopólio acarretaria em uma queda de preço dos medicamentos, sendo o segundo efeito desejado. Por sua vez, a queda dos preços permitiria que uma nova onda de consumidores, antes sem acesso aos medicamentos devido aos preços elevados, passasse a fazer parte do mercado, resultando na expansão da quantidade de medicamentos vendidos.

Tendo em vista esses três efeitos, procura-se analisar o grau de sucesso da política de medicamentos genéricos através da verificação de sua relação com o impacto sobre a estrutura de mercado, sobre o preço ao consumidor final e sobre a quantidade vendida de medicamentos.

Para a política atingir a meta da primeira etapa da cadeia, o efeito sobre a estrutura de mercado precisa ser de redução do HHI, levando-se à conclusão de que os medicamentos genéricos poderiam estar substituindo produtos de referência ou similares.

No tocante ao impacto sobre os preços ao consumidor final, espera-se que, na medida em que os produtos genéricos entrem no mercado com preços mais baixos que os de referência, o preço médio dos medicamentos sofra uma queda.

Uma vez verificada a redução do HHI e a queda do preço médio dos medicamentos, o foco passa a ser o efeito sobre a quantidade de medicamentos vendida.

$4 \quad$ Ver Mendonça (2010).

5 O cálculo do $\mathrm{HHI}$ é definido por $\mathrm{HHI}=\left(\Sigma \mathrm{si}^{2}\right) 10000$, em que si é a razão das vendas da empresa $i$ pelo total de vendas do mercado (parcela de mercado da empresa i) (VARIAN, 2003). Ver Mendonça (2010). 
Caso o efeito não seja verificado, o principal objetivo da política dos medicamentos genéricos, que é o aumento do acesso da população aos medicamentos, não estará sendo atendido.

\subsubsection{Críticas e Possíveis Vieses}

Primeiramente, todos os trabalhos auxiliados pela metodologia de classificação por mercado relevante merecem uma ressalva pela arbitrariedade desse processo (GAMA; CAVALIERI, 2006). Apesar de não ser uma regra, a falta de consenso em torno da classificação do mercado relevante oferece margem à crítica de que o resultado poderia ser diferenciado em função da escolha feita.

A presente dissertação está suscetível a essa crítica, que procurou minimizá-la pela assistência de profissionais da área de saúde. Ao mesmo tempo, entende-se que, por trás da metodologia de mercado relevante, vários resultados quantitativos podem ser auferidos em benefício do tema analisado. Portanto, essa primeira crítica deve ser colocada em segundo plano para que a direção da política de medicamentos genéricos possa ser contemplada.

Em relação aos dados, como já foi exposto, diferenças nas apresentações, como a forma farmacêutica e a concentração do princípio ativo, não foram levadas em consideração por aumentarem demasiadamente a complexidade do estudo. Por outro lado, outra fonte de viés não foi negligenciada: foram excluídos da análise medicamentos de venda exclusiva para o setor público. Essa escolha se deu em virtude da intenção de se captar a importância dos medicamentos genéricos diretamente no setor varejista e também para não cair sobre um viés relacionado a prováveis diferenças nas taxas de crescimento dos diferentes tipos de demanda.

Como a demanda governamental pode ser deslocada de maneira mais imediata para o medicamento genérico, devido a não intermediação dos médicos e à obrigatoriedade de seguir a forma de prescrição adotada pelos hospitais, sua taxa de crescimento dependerá apenas do surgimento de genéricos em novos princípios ativos ou da própria demanda pelo princípio ativo como um todo. Enquanto isso, na demanda privada, devido à intermediação dos médicos, não há obrigatoriedade na adesão imediata aos genéricos, portanto a taxa de crescimento deverá ser lenta inicialmente, crescendo paulatinamente.

O viés da taxa de crescimento também serviu para a escolha do período a ser analisado. Considerando o prazo de três anos adequado para estabilizar o salto inicial do uso dos medicamentos genéricos, preferiu-se iniciar em 2003 para que os anos iniciais não prejudicassem a análise, podendo indicar, em um dos cenários, uma adesão à política de medicamentos genéricos mais rápida que na realidade. 
Discorrido sobre os itens abordados nas seções anteriores, passa-se a expor os resultados obtidos pelos testes empíricos realizados na mesma ordem em que foram apresentadas as hipóteses.

\section{$2.4 \mathrm{O}$ Impacto sobre a Estrutura do Mercado}

O primeiro teste a ser realizado diz respeito ao impacto da introdução dos medicamentos genéricos sobre a estrutura do mercado farmacêutico brasileiro. Espera-se verificar que a entrada dos medicamentos genéricos tenha contribuído para a diminuição da concentração na indústria. Para isso, começa-se por uma análise da evolução do HHI entre janeiro de 2003 e dezembro de 2007, conforme pode ser observado na Tabela 6 .

Tabela 6 - Faixas de concentração dos mercados relevantes entre 2003 e 2007

\begin{tabular}{|c|c|c|c|c|c|c|c|c|}
\hline \multirow[b]{2}{*}{$\begin{array}{c}\text { Faixas de } \\
\text { Concentração }\end{array}$} & \multirow{2}{*}{$\begin{array}{c}\text { Janeiro de } \\
\text { Número de } \\
\text { Mercados } \\
\text { Relevantes }\end{array}$} & \multicolumn{3}{|l|}{2003} & \multicolumn{4}{|c|}{ Dezembro de 2007} \\
\hline & & $\%$ & $\begin{array}{c}\text { Soma de } \\
\text { Vendas } \\
\text { (R\$1.000) }\end{array}$ & $\%$ & $\begin{array}{l}\text { Número de } \\
\text { Mercados } \\
\text { Relevantes }\end{array}$ & $\%$ & $\begin{array}{c}\text { Soma de } \\
\text { Vendas } \\
\text { (R\$1.000) }\end{array}$ & $\%$ \\
\hline $0-1.000$ & 17 & 37,8 & 174.506 & 71,7 & 20 & 44,4 & 300.658 & 81,5 \\
\hline $1.000-1.800$ & 9 & 20 & 29.680 & 12,2 & 8 & 17,8 & 34.539 & 9,4 \\
\hline $1.800-9.999$ & 18 & 40 & 39.212 & 16,1 & 16 & 35,6 & 33.343 & 9,1 \\
\hline 10.000 & 1 & 2,2 & 113 & 0 & 1 & 2,2 & 184 & 0 \\
\hline Total & 45 & 100 & 243.511 & & 45 & 100 & 368.724 & 100 \\
\hline
\end{tabular}

Fonte: Elaboração própria a partir de dados da Anvisa.

Os dados apresentados sugerem que houve uma diminuição da concentração entre 2003 e 2007, sem apontar a causa de tal movimento. A primeira faixa de concentração é classificada como competitiva. Nessa categoria, foi observado o aumento do número de mercados relevantes, o que colabora com a hipótese inicial proposta. Em janeiro de 2003, os mercados relevantes enquadrados como competitivos somavam $37,8 \%$ do total e passaram para $44,4 \%$ em dezembro de 2007 , assumindo a maior participação entre as faixas de concentração.

Em relação às outras faixas de concentração, observa-se que um mercado relevante de concentração moderada provavelmente migrou para a categoria de mercado competitivo e que dois de alta concentração também tiveram diluição do seu HHI. Apenas o monopólio não sofreu alteração, seja por uma proteção patentearia ou por outras barreiras que impediram a entrada de novos concorrentes.

Apesar dos mercados relevantes competitivos não serem a maioria desde o começo do período analisado, em termos de soma de vendas sempre tiveram a maior participação, deixando indícios de uma relação inversamente proporcional entre o grau de concentração e o tamanho do mercado relevante. Ao mesmo tempo, a presença de medicamentos genéricos também parece ser mais comum em mercados menos concentrados, e a constatação desse fato contribuiria de forma definitiva para a apro- 
vação da hipótese de que a introdução dos medicamentos genéricos contribuiu para a redução da concentração no mercado farmacêutico.

Um resultado que pode ser deduzido através da observação da Tabela 6 é que o crescimento das vendas foi impulsionado pelo segmento mais competitivo. Enquanto as vendas totais do mercado analisado cresceram $51,4 \%$, passando de $R \$ 243,5$ milhões para $\mathrm{R} \$ 368,7$ milhões, as vendas da primeira faixa de concentração cresceram $72,3 \%$ e totalizaram $\mathrm{R} \$ 300,7$ milhões, ou $81,5 \%$ do total vendido. O segmento monopolista foi o segundo maior em crescimento, com $62,8 \%$, mas as vendas de $\mathrm{R} \$ 184$ mil não chegaram nem a $0,1 \%$ do total, portanto praticamente não exerceram influência sobre o crescimento total. Já os outros segmentos impediram que o crescimento total tivesse melhor desempenho. A segunda faixa de concentração cresceu apenas 16,4\% e a terceira apresentou queda de $15 \%$ nas vendas. Ambos os resultados podendo ser justificados pelos processos migratórios apontados anteriormente.

No tocante ao número de genéricos encontrados na amostra de mercado analisado, ao todo foram classificados entre os mercados relevantes 1.858 medicamentos genéricos, distribuídos conforme mostra a Tabela 7. Nota-se uma grande aglomeração de medicamentos genéricos na primeira faixa de concentração e sua subsequente diminuição em faixas de concentração mais elevada, como sugere a intuição de que a presença dos medicamentos genéricos influencia a competitividade dos mercados relevantes. Em números, 1.569 medicamentos genéricos, ou 84,4\% do total de 1.858, encontram-se na faixa de HHI mais baixo. Na faixa de concentração seguinte, esse número cai para 193, respondendo por 10,4\%, e, na terceira faixa, apenas 96 medicamentos genéricos são contabilizados, pouco mais de 5\%. A faixa de monopólio, logicamente, não apresenta medicamentos genéricos, até porque se é fruto de uma proteção por patente, os medicamentos genéricos ainda não podem atuar nesse segmento.

Tabela 7 - Presença dos medicamentos genéricos por faixas de concentração dos mercados relevantes acumulada entre 2003 e 2007

\begin{tabular}{ccc}
\hline $\begin{array}{c}\text { Faixas de } \\
\text { Concentração }\end{array}$ & $\begin{array}{c}\text { Número de } \\
\text { Medicamentos } \\
\text { Genéricos }\end{array}$ & Participação (\%) \\
\hline $0-1.000$ & 1569 & 84,4 \\
$1.000-1.800$ & 193 & 10,4 \\
$1.800-9.999$ & 96 & 5,2 \\
10.000 & 0 & 0 \\
\hline Total & 1858 & 100 \\
\hline
\end{tabular}

Fonte: Elaboração própria a partir de dados da Anvisa. 
Não se limitando somente à observação empírica para testar a hipótese de o tamanho do mercado e a presença de medicamentos genéricos influenciarem a estrutura produtiva do mercado farmacêutico, foi construída uma equação de mínimos quadrados ordinários da seguinte forma (HILL et al., 2000):

$$
Y_{i}=\beta_{0}+\beta_{1} X_{1 i}+\beta_{2} X_{2 i}+\varepsilon_{i}
$$

Em que: $Y_{i}$ é a variável dependente logaritmo natural do grau de concentração do mercado relevante i; $\beta_{0}$ é o intercepto; $\beta_{1}$ e $\beta_{2}$ são, respectivamente, os parâmetros para as variáveis independentes logaritmo natural das vendas, representado por $X_{1 i}$, e o número de medicamentos genéricos, $X_{2 i} ; \varepsilon_{i}$ é o erro.

Os dados selecionados para esse teste foram as observações do último período da série, dezembro de 2007, por ser o período em que a política de genéricos estaria mais madura, maximizando as chances de se obter uma participação contundente dos medicamentos genéricos nos mercados relevantes em que estivessem presentes.

A seguir, os resultados do teste de identificação das influências para a diminuição do grau de concentração são mostrados através da Tabela 8.

Tabela 8 - Regressão de mínimos quadrados ordinários - variável dependente: logaritmo natural do $\mathrm{HHI}$

\begin{tabular}{cc}
\hline Variável & Valor \\
\hline Constante & $4,0716^{* *}$ \\
& $(14,70080)$ \\
Ln Vendas & $-0,1157$ \\
& $(-2,5079)$ \\
Número de Genéricos & $-0,0067^{* *}$ \\
& $(-7,4190)$ \\
\hline Estatística & \\
\hline $\mathbf{R}^{2}$ & 0,7839 \\
$\mathbf{F}$ & $76,1678^{* *}$ \\
$\mathbf{N}$ & 45 \\
\hline
\end{tabular}

Fonte: Elaboração própria.

Nota: Estatística t entre parênteses; "significativo a 5\%; * "significativo a 1\%. 
A equação tem boa adequação, pois apresenta estatística $F$ significativa a 1\% e $R^{2}$ de 0,7839 , o que é satisfatório para regressões de corte horizontal. A estatística $F$ diz respeito ao teste de hipótese de que todos os coeficientes da regressão, sem contar com a constante, são nulos. Ao se obter uma estatística $F$ significativa, a hipótese nula é rejeitada, validando o teste, mesmo quando todas as estatísticas $t$ individuais são insignificantes. Já o $\mathrm{R}^{2}$ mede a proximidade entre os valores estimados e os observados da variável dependente dentro da amostra utilizada para estimar a regressão, ou seja, é uma medida de sucesso da estimativa.

Em relação ao tamanho do mercado, definido pelas vendas totais do mercado relevante, seu coeficiente está negativamente correlacionado ao grau de concentração como se previa, assim como a quantidade de medicamentos genéricos comercializados, ambos significativos. Em outras palavras, o teste concluiu que o grau de concentração diminui quanto maior for o nível de vendas do mercado e quanto maior for a presença de medicamentos genéricos.

Como essas variáveis não foram testadas em meio a séries temporais, não existe o argumento de que essas relações possam ser espúrias, ${ }^{6}$ apesar do fato de se trabalhar com séries temporais também não implicar obrigatoriamente nesse problema. No entanto, um artifício que se mostrou necessário foi a aplicação do logaritmo natural nas variáveis $\mathrm{HHI}$ e vendas para a redução do desvio padrão dos coeficientes. Sem essa técnica, os coeficientes perderiam sua significância, o que os deixariam sem consistência para afirmação de suas importâncias, mesmo sendo um resultado intuitivo. O mesmo expediente não foi utilizado para a variável número de genéricos porque acarretaria a perda das observações em que estes não estivessem presentes, e isso iria contra o objetivo do teste.

Outras formas de equação foram testadas de maneira a incluir a quantidade de medicamentos éticos vendidos e o número de princípios ativos dentro do mercado relevante, mas nenhuma outra variável contribuiu para deixar o resultado apresentado acima mais robusto. Portanto, deu-se preferência a essa forma mais simples, de modo a atender o critério de parcimônia ${ }^{7}$ dos modelos econométricos.

Verificada a relação existente entre a presença dos medicamentos genéricos e o grau de concentração do mercado relevante, o próximo passo a se seguir é testar se a intuição também se encontra com os dados empíricos no tocante ao preço.

6 Uma relação espúria apresenta resultados significantes a partir de dados não relacionados, ou seja, a significância da regressão é falsa (HILL et al., 2000).

7 O critério segue o conceito geral de "quanto menos, melhor" e sua utilização não se limita somente à economia. Pelo conceito aplicado especificamente na econometria, para a melhor identificação da relação entre as variáveis é preferível um modelo menor ao invés de um modelo mais complexo. No entanto, atenta-se para o trade-off entre a simplicidade e a perda de poder explicativo do modelo. O critério de seleção deve seguir o bom senso, pois não adianta um modelo simples identificar a relação entre suas variáveis, mas pouco explicar o contexto de tal relação, assim como não adianta um modelo complexo reproduzir a realidade com perfeição e ao mesmo tempo não permitir o entendimento de sua engenharia (HILL et al., 2000). 


\subsection{Ompacto sobre os Preços dos Medicamentos}

Segundo a hipótese levantada anteriormente, a entrada dos medicamentos genéricos é capaz de causar a diminuição do grau de concentração em um mercado relevante, e um efeito secundário seria a diminuição do preço médio dos medicamentos como um todo nesse segmento, em decorrência da diminuição do poder de mercado dos fabricantes de medicamentos.

O primeiro efeito foi testado e verificou-se que a concentração dos mercados relevantes em que os medicamentos genéricos tiveram acesso de fato reduziu. Seguindo a cadeia de raciocínio, o teste sobre o segundo efeito, o da redução dos preços, começa com a observação da evolução dos preços reais médios dos medicamentos para o período analisado. De acordo com os dados trabalhados, o preço médio dos medicamentos diminuiu entre janeiro de 2003 e dezembro de 2007, como pode ser observado no Gráfico 1, para os 45 mercados relevantes analisados.

Essa primeira análise aponta para uma redução média dos preços dos medicamentos da amostragem de $0,2 \%$ ao mês, em consequência do aumento nominal de $0,3 \%$ ao mês ser menor que a inflação média de $0,5 \%$ ao mês para o mesmo período, medido pelo Índice de Preços ao Consumidor Amplo (IPCA) do Instituto Brasileiro de Geografia e Estatística (IBGE). À primeira vista, a redução do preço médio é positiva para a hipótese levantada. Entretanto, como o indicador não considera as especificidades de cada mercado relevante, uma análise mais detalhada se mostra necessária para inferir sobre o sucesso do programa de medicamentos genéricos.

Gráfico 1 - Preço real médio dos medicamentos entre 2003 e 2007

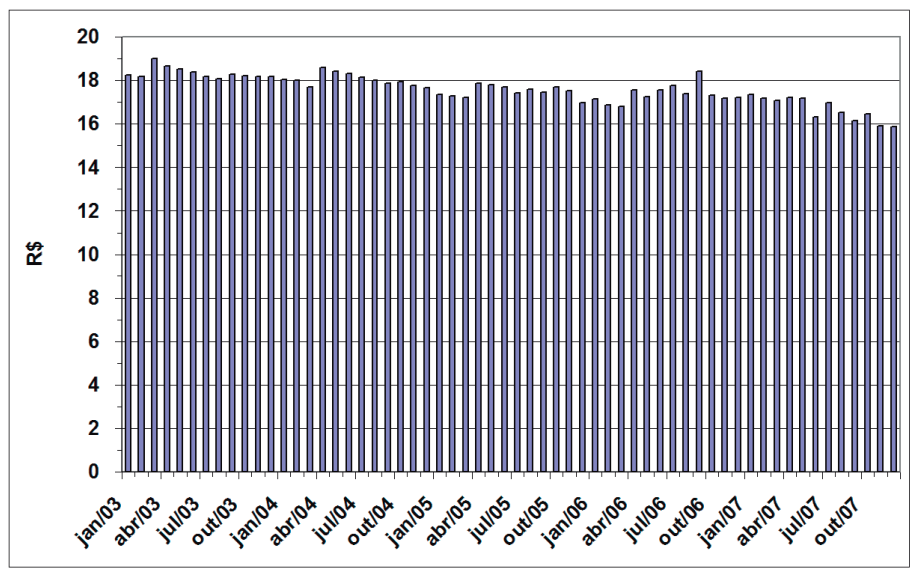

Fonte: Elaboração própria a partir de dados da Anvisa. 
Cada faixa de concentração deve apresentar um comportamento de preço. Os mercados classificados como competitivos devem ter os menores preços e os mais concentrados devem ficar com os maiores. Por essa lógica, se os mercados concentrados possuírem maior poder de mercado para elevar os preços, em comparação à força que os mercados competitivos teriam para baixá-los, o preço médio se elevaria, o que não se configura pelo comportamento do Gráfico 1.

Para procurar identificar possíveis efeitos da entrada dos medicamentos genéricos sobre a variação dos preços dos medicamentos, principalmente sobre o medicamento de referência, foram adotados alguns procedimentos encontrados na literatura. Por exemplo, o de Caves et al. (1991), que analisa a variação do logaritmo da razão entre o preço médio dos medicamentos genéricos e o preço médio dos medicamentos de referência em uma regressão em painel com efeitos fixos.

Esse procedimento visaria capturar um tipo de estratégia de preços que as firmas líderes de mercado parecem adotar com a entrada dos medicamentos genéricos. O modelo sugere que as líderes diferenciam seus preços de acordo com os consumidores envolvidos nas vendas. Em particular, em segmentos com menor elasticidade a mudanças dos preços, as empresas produtoras de medicamentos de referência elevariam seus preços e deixariam os segmentos com maior elasticidade para os medicamentos genéricos.

Tabela 9 - Regressão em painel com efeitos fixos - variável dependente: logaritmo natural da razão entre o preço médio dos medicamentos genéricos e dos de referência

\begin{tabular}{cc}
\hline Variável & Valor \\
\hline Constante & $8,0510^{*}$ \\
& $-42,365$ \\
Ln HHI & $-0,3751^{*}$ \\
& $(-25,0992)$ \\
Ln Vendas dos Genéricos & $-0,0033$ \\
& $(-0,6950)$ \\
Ln Vendas Totais & $-0,2227^{*}$ \\
& $(-29,8274)$ \\
\hline Estatística & \\
\hline $\mathrm{R}^{2}$ & 0,2839 \\
$\mathrm{~F}$ & $319,3352^{*}$ \\
$\mathrm{~N}$ & 2421 \\
\hline
\end{tabular}

Fonte: Elaboração própria.

Nota: Estatística t entre parênteses; "significativo a 1\%. 
O resultado do teste é apresentado na Tabela 9, e, conforme pode ser verificado, o grau de sucesso da equação, denominado pelo $R^{2}$, foi de pouco mais de $28 \%$. Além disso, o coeficiente da variável de vendas dos medicamentos genéricos não retornou um resultado com nível de significância suficiente, portanto não tem validade estatística. Por outro lado, a estatística $F$ e as variáveis de concentração e vendas totais obtiveram resultados com implicações positivas para a análise. Os coeficientes negativos e estatisticamente significativos para esse modelo indicam que a estratégia das empresas estaria distanciando os preços e que provavelmente algum tipo de segmentação de mercado estaria se formando.

Em relação ao baixo poder explicativo da equação e ao nível de significância insuficiente para a variável vendas dos genéricos, uma característica que não deve ser esquecida sobre a política de medicamentos genéricos é que sua atuação é indireta sobre o preço do mercado. A administração da política de medicamentos genéricos empreendida pelo Ministério da Saúde e pela Anvisa induz que os medicamentos genéricos sejam postos no mercado a preços $35 \%$ inferiores aos preços dos medicamentos de referência. Portanto, em um cenário de incremento de preços, o preço referencial estabelecido pelas autoridades sanitárias também sofrerá o mesmo impacto sem uma medida simultânea que o desvie desse comportamento.

Além disso, lembra-se que a demanda tende a ser inelástica a variações de preço em função da essencialidade do produto e que a decisão de consumo não é feita diretamente pelo consumidor, e sim pelo prescritor. Isso favorece as marcas existentes que se aproveitam da assimetria de informação e da política de marketing para convencer os prescritores a manterem a medicação com eficácia reconhecida apesar de seu preço. Por sua vez, o prescritor sem a informação completa sobre a eficácia do medicamento genérico e sob o receio de comprometer a recuperação de seu paciente acaba por atender ao pleito dos medicamentos de marca.

Uma limitação importante do modelo também pode estar contribuindo para a obtenção de um resultado estatisticamente falho. Aponta-se como outra possível causa do problema a forma como foram classificados os dados. A agregação de todas as formas de apresentação sobre o mesmo mercado relevante para simplificar a classificação pode ter prejudicado o resultado do teste. A diferença entre os preços de medicamentos injetáveis e comprimidos simples, ou xaropes e pomadas, em alguns casos, é muito grande, e um refinamento maior, com a distinção dessas formas de apresentação, seria mais adequado. Entretanto essa decisão não foi tomada, pois se entendeu que não haveria tempo, nem conhecimento técnico suficiente para a medida.

Apesar da falta de um resultado estatisticamente significativo para a variável com maior apelo à política de medicamentos genéricos, acredita-se que não ocorra uma quebra na cadeia de efeitos a serem conseguidos pela introdução dos medicamentos genéricos, pois a redução dos preços no mercado farmacêutico foi 
verificada. A seguir, parte-se para o teste dos impactos sobre a quantidade comercializada, pois esse é o principal foco da política de medicamentos genéricos.

\subsection{O Impacto sobre a Quantidade Comercializada}

Ao se analisar o impacto sobre a quantidade comercializada de medicamentos, o primeiro ponto que deve ser examinado é como o mercado de medicamentos se comportou durante o período.

Segundo os dados da Tabela 10, entre 2003 e 2007, os 94 princípios ativos que formaram a fatia do mercado estudado registraram uma expansão de unidades vendidas de $18,9 \%$, ou uma média de crescimento de $4,4 \%$ ao ano. Tal desempenho teve forte relação com o crescimento das vendas dos medicamentos genéricos, que no mesmo período alcançou $96,0 \%$, ou uma média de $18,4 \%$ ao ano.

Tabela 10 - Evolução das quantidades vendidas de medicamentos por tipo entre 2003 e 2007

\begin{tabular}{lrrrrrr}
\hline & $\mathbf{2 0 0 3}$ & $\mathbf{2 0 0 4}$ & $\mathbf{2 0 0 5}$ & $\mathbf{2 0 0 6}$ & $\mathbf{2 0 0 7}$ & Acumulado \\
\hline Genéricos & 73.817 .154 & 90.563 .149 & 107.721 .975 & 127.935 .595 & 144.699 .901 & 544.737 .774 \\
Participação & $24,25 \%$ & $27,85 \%$ & $31,95 \%$ & $36,54 \%$ & $39,99 \%$ & $32,45 \%$ \\
Crescimento & & $22,69 \%$ & $18,95 \%$ & $18,76 \%$ & $13,10 \%$ & $96,02 \%$ \\
& & & & & & \\
Marca & 74.303 .592 & 80.562 .407 & 85.972 .268 & 84.683 .701 & 89.353 .782 & 414.875 .750 \\
Participação & $24,41 \%$ & $24,77 \%$ & $25,50 \%$ & $24,18 \%$ & $24,69 \%$ & $24,71 \%$ \\
Crescimento & & $8,42 \%$ & $6,72 \%$ & $-1,50 \%$ & $5,51 \%$ & $20,25 \%$ \\
& & & & & & \\
Referência & 156.062 .316 & 153.875 .240 & 143.215 .980 & 137.315 .294 & 127.575 .616 & 718.044 .446 \\
Participação & $51,26 \%$ & $47,31 \%$ & $42,48 \%$ & $39,21 \%$ & $35,26 \%$ & $42,77 \%$ \\
Crescimento & & $-1,40 \%$ & $-6,93 \%$ & $-4,12 \%$ & $-7,09 \%$ & $-18,25 \%$ \\
Similar & 246.311 & 229.582 & 215.388 & 235.992 & 225.268 & 1.152 .541 \\
Participação & $0,08 \%$ & $0,07 \%$ & $0,06 \%$ & $0,07 \%$ & $0,06 \%$ & $0,07 \%$ \\
Crescimento & & $-6,79 \%$ & $-6,18 \%$ & $9,57 \%$ & $-4,54 \%$ & $-8,54 \%$ \\
\hline
\end{tabular}

Total

$\begin{array}{lllllll}304.429 .373 & 325.230 .378 & 337.125 .611 & 350.170 .582 & 361.854 .567 & 1.678 .810 .511\end{array}$

Fonte: Elaboração própria a partir de dados da Anvisa. 
Em 2003, os medicamentos de referência detinham 51,3\% de participação, frente a $24,2 \%$ de participação dos medicamentos genéricos. Em 2007, os medicamentos genéricos passaram a assumir a maior participação, com aproximadamente $40,0 \%$, enquanto que os medicamentos de referência ocupavam $35,3 \%$ das unidades vendidas. No entanto, medicamentos de referência são medicamentos de marca, então, somando os dois tipos, os medicamentos genéricos voltam a não ser as unidades preponderantes no mercado.

A forte presença dos medicamentos genéricos no segmento estudado precisa ser relevada. Como o resultado com os preços dos medicamentos não foi inteiramente conclusivo, fica a hipótese de poder ter ocorrido uma mudança no comportamento das firmas estabelecidas, no sentido de ceder essa parcela de mercado para os genéricos. Nesse caso, as empresas líderes estariam reduzindo os investimentos na divulgação de seus produtos para dirigir seus recursos à fabricação de novos medicamentos, que estariam protegidos por patentes e, consequentemente, da concorrência dos medicamentos genéricos.

Em todo caso, espera-se contribuir para a compreensão do efeito da introdução dos medicamentos genéricos sobre as unidades vendidas, tentando diferenciar os mercados de acordo com a participação total dos genéricos. Para isso, elaborou-se uma regressão com dados em painel com efeitos fixos sob a forma apresentada a seguir:

$$
Y_{i t}=\beta_{0}+\beta_{1} X_{1 i t}+\beta_{2} X_{2 i t}+\beta_{3} X_{3 i t}+\varepsilon_{i t}
$$

onde $Y_{\text {it }}$ é a variável dependente logaritmo natural do número de unidades vendidas do mercado relevante i no período $t ; \beta_{0}$ é o intercepto; $\beta_{1}, \beta_{2}$ e $\beta_{3}$ são, respectivamente, os coeficientes para as variáveis independentes logaritmo natural do grau de concentração do mercado relevante, representado por $X_{\text {Iit }}$, logaritmo natural das vendas, em valor, dos medicamentos genéricos, $X_{2 i t}$, e logaritmo natural das vendas totais, $X_{3 \mathrm{it}}$; e $\varepsilon_{i}$ é o erro.

Na regressão aplicou-se o logaritmo natural para reduzir o desvio padrão dos coeficientes e ganhar robustez nos resultados. Em seguida, foi realizado o teste de raiz unitária de Dickey-Fuller-Fisher aumentado ${ }^{8}$ nas variáveis transformadas, a procura de séries não estacionárias, o que, dessa vez por estar se trabalhando em função do tempo, poderia resultar em uma relação espúria. Atentando-se para esses cuidados, os resultados são apresentados na Tabela 11.

$8 \quad$ Ver Mendonça (2010). 
Tabela 11 - Regressão em painel com efeitos fixos - variável dependente: logaritmo natural da quantidade vendida

\begin{tabular}{cc}
\hline Variável & Valor \\
\hline Constante & $-2,1102^{*}$ \\
Ln HHI & $(-18,8692)$ \\
& $0,2251^{*}$ \\
Ln Vendas dos Genéricos & $(-11,2185)$ \\
& $0,0263^{*}$ \\
Ln Vendas Totais & $(-6,4622)$ \\
& $1,0315^{*}$ \\
Estatística & $(-102,0352)$ \\
\hline R & 0,89 \\
F & $7266,0558^{*}$ \\
\hline
\end{tabular}

Fonte: Elaboração própria.

Nota: Estatística t entre parênteses; *significativo a 1\%.

Os resultados parecem ser positivos para a política de medicamentos genéricos. $O R^{2}$ indica boa adequação, o $F$ é significante e todas as variáveis explicativas apresentam sinal positivo e são estatisticamente significativas a 1\%. Em especial, o coeficiente da variável de vendas dos genéricos implica na interpretação desejada de que os medicamentos genéricos conseguiram ser inseridos no mercado com um resultado positivo sobre a quantidade comercializada, portanto a política tem logrado êxito com esse mérito. Por outro lado, o coeficiente da variável de concentração pode causar desconforto por ser positivo. Uma possível interpretação para essa informação diz respeito à possibilidade das firmas estabelecidas estarem adotando a estratégia de produzir seus próprios medicamentos genéricos para competir nesse segmento, como de fato pode ser observado no mercado farmacêutico brasileiro.

Em relação à econometria utilizada, além do modelo de efeitos fixos, dados em painel também podem ser modelados sob efeitos aleatórios. Entretanto, o modelo fixo se mostrou superior, nesse caso, em virtude da homogeneidade das estimativas de correlação, sendo conferido pelo teste de Hausman (WOOLDRIDGE, 2002).

9 O teste de Hausman estabelece como hipótese nula, em um caso de efeitos fixos, que modelos de efeitos aleatórios são consistentes e, como hipótese alternativa, que não são consistentes. Se houver rejeição da hipótese nula, a conclusão será que o modelo de efeitos fixos oferece resultados mais consistentes do que os do modelo de efeitos aleatórios. 
Novos modelos com novas variáveis sempre deveriam surgir para explicar melhor alguns resultados encontrados. Contudo, a grande contribuição deste trabalho é poder avaliar a política dos medicamentos genéricos durante um longo prazo, alcançando boa adequação. Com o passar do tempo, espera-se que novas análises possam aparecer, visando em última instância à orientação tanto da população quanto dos próprios policy makers.

\section{Considerações Finais}

Este trabalho permitiu verificar que, entre 2003 e 2007, a concentração do mercado diminuiu e a estimativa do modelo proposto retornou um resultado positivo para a hipótese de aumento da concorrência. Como foi testado, quando a participação dos medicamentos genéricos foi maior, menor foi o grau de concentração dos mercados relevantes. Ao mesmo tempo, outro resultado observado foi que existe uma relação negativa entre as vendas totais do mercado farmacêutico e sua concentração, sugerindo o fim da hegemonia nas vendas pelos medicamentos que detêm maior parcela do mercado.

Em relação ao comportamento dos preços dos medicamentos, o modelo retornou um baixo poder explicativo. A justificativa para o resultado débil pode ser encontrada no próprio caráter da política de diminuição de preços via introdução de medicamentos genéricos, que é uma forma indireta de controlar os preços, uma vez que atua sobre a concorrência e não sobre as características que definem os preços diretamente, como, por exemplo, o mark-up das firmas. Entretanto, o modelo adotado sugeriu que pode estar ocorrendo uma forma de segmentação de mercado em função do aumento da distância entre os preços dos medicamentos genéricos e dos de referência.

Outra alternativa para o fraco desempenho do modelo que visou captar o comportamento dos preços pode ser a forma em que foram classificados os dados, sendo uma crítica à forma de agregação das diferentes apresentações farmacêuticas. Nesse caso, a contribuição deste artigo para a questão sobre o preço dos medicamentos acaba sendo uma proposta de mudança na forma de classificar as apresentações para testar em até que ponto isso pode afetar o teste da hipótese.

O levantamento da amostra em termos de mercado relevante também contribui para a limitação do poder explicativo do modelo, pois a política de medicamentos genéricos tem como alvo central um medicamento de referência de um determinado princípio ativo. Contudo, pela definição dada de mercado relevante, a realização dos testes levando-se em conta os princípios ativos também poderia limitar o modelo no sentido de se superestimar o poder de monopólio das firmas por tornar a definição de concorrência entre firmas muito estreita. 
O resultado sobre a variação da quantidade comercializada superou as expectativas. A regressão retornou um $R^{2}$ adequado para cortes longitudinais e os coeficientes utilizados indicaram que a presença dos medicamentos genéricos favoreceu o volume de vendas, significando que houve uma melhoria do acesso aos medicamentos para a população. Atribui-se a esse resultado positivo o período escolhido para a análise, pois se atentou para que o período refletisse condições de maturidade da política de medicamentos genéricos, e não o início com taxas de crescimento exponenciais.

Em suma, os resultados são positivos para a política de medicamentos genéricos, mas indica que ainda há o que ser feito. Em comparação com o resultado norte-americano, o mercado de medicamentos genéricos brasileiro mostra que ainda é capaz de se expandir, pois o mercado americano, mesmo após 25 de experiência, ainda não encontrou seu ponto de saturação. Sendo assim, acredita-se que o nível de saturação do mercado brasileiro só se aproxime ao fim da próxima década.

\section{Referências}

ACADEMIA BRASILEIRA DE CIÊNCIAS. Medicamentos a partir de planos medicinais no Brasil. Rio de Janeiro: Academia Brasileira de Ciências, 1998.

ASSOCIAÇÃO BRASILEIRA DA INDÚSTRIA DE MEDICAMENTOS ISENTOS DE PRESCRIÇÃO. Dados mercadológicos. São Paulo. Disponível em: < http://www.abimip.org.br/dados. htm>. Acesso em: 12 nov. 2009.

BAIN, J. S. Industrial organisation. New York: John Wiley E Sons, 1959.

BAUMOL, W. J. Contestable markets: an uprising in the theory of industrial structure. American Economic Review, Pittsburgh, USA, n. 72, p. 1-15, 1982.

BERMUDEZ, J. A. Z. et al. O Acordo Trips da OMC e a proteção patentária no Brasil: mudanças recentes e implicações para a produção local e o acesso da população aos medicamentos. Rio de Janeiro: ENSP/Fiocruz, 2000.

BERNDT, E. R. The U.S. pharmaceutical industry: why major growth in times of cost containment? Health Affairs, Bethesda, USA, n. 20, v. 2, p. 100-114, 2001.

BRASIL. Ministério da Saúde. Indicadores e dados básicos. Brasília, DF. Disponível em: <http://tabnet.datasus.gov.br/cgi/tabcgi.exe?idb2008/c04.def>. Acesso em: 25 out. 2009.

CAVES, R. E.; WHINSTON, M. D.; HURWITZ, M. A. Patent expiration, entry, and competition in the U.S. pharmaceutical industry. Brookings Papers on Economic Activity, Washington, DC, USA, p. 1-66, 1991.

DASGUPTA, P.; STIGLITZ, J. E. Uncertainty, industrial structure and the speed of RED. Bell Journal of Economics, n. 11, p. 1-28, 1980. 
DAVIES, S. et al. Economics of industrial organisation. New York: Longman Group, 1988.

EUROPEAN FEDERATION OF PHARMACEUTICAL INDUSTRIES AND ASSOCIATIONS. The pharmaceutical industry in figures 2009. Disponivel em: <http://www.efpia.eu/content/ default.asp?PageID=559EDDocID=4883 > . Acesso em: 12 nov. 2009.

GAMA, M. M.; CAVALIERI, M. A. R. Crítica à avaliação quantitativa do efeito unilateral de um ato de concentração. Belo Horizonte: UFMG/Cedeplar, 2006. (Texto para Discussão n. 295).

GRABOWSKI, H. The effect of pharmacoeconomics on company research and development decisions. PharmacoEconomics, n. 11, v. 5, p. 389-397, 1997.

GRABOWSKI, H.; VERNON, J. Longer patents for increased generic competition in the U.S.: the Waxman-Hatch Act after one decade. Pharmacoeconomics, n. 10, v. 2, p. 110-123, 1996.

HILL, R. C.; GRIFFITHS, W. E.; JUDGE, G. G. Econometria. São Paulo: Saraiva, 2000.

HOTELLING, H. Stability in competition. Economic Journal, n. 39, p. 41-57, 1929.

INTERNATIONAL MEDICAL STATISTICS. 2007 Top-line insustry data. Disponível em: <http://www.imshealth.com/vgn-ext-templating/v/index.jsp?vgnextoid=67a89df4609e9110 VgnVCM10000071812ca2RCRDE3cpsextcurrchannel=1 >. Acesso em: 31 dez. 2008.

.IMS retail drug monitor December 2007. Disponível em: <http://www1.imshealth. com/web/content/0,3148,64576068_63872702_70260998_83746585,00.html>. Acesso em: $31 \mathrm{dez} .2008$.

PHARMACEUTICAL RESEARCH AND MANUFACTURERS OF AMERICA. Pharmaceutical industry profile 2008. Washington, DC, USA: PhRMA, 2008.

SARMIENTO, A. Z. Alternative drug pricing policies in the Americas. Genova: World Health Organization, 1995. (Health Economics and Drugs, DAP Series, n. 1).

SCHELLING, T. C. The strategy of conflict. Havard: Havard University Press, 1960.

SCHERER, F. M. Industrial market structure and economic performance. Chicago: Rand McNally, 1970.

SCHUMPETER, J. A. Capitalism, socialism and democracy. London: Unwin, 1943.

SHAKED, A.; SUTTON, J. Product differentiation and industrial structure. Journal of Industrial Economics, n. 36, p. 131-146, 1987.

SMITH, A. A riqueza das nações. Curitiba: Juruá, 2006.

UNITED STATES OF AMERICA. Department of Justice. Sherman Antitrust Act. Disponível em: <http://www.usdoj.gov/atr/public/divisionmanual/chapter2.pdf>. Acesso em: 25 out. 2009 .

UNIVERSIDADE FEDERAL DO RIO DE JANEIRO. Instituto de Economia. Diagnóstico da indústria farmacêutica brasileira. Rio de Janeiro: IE/UFRJ, 2002. Mimeografado. 
VARIAN, H. R. Microeconomia: princípios básicos. Rio de Janeiro: Campus, 2003.

VON STACKELBERG, H. The theory of the market economy. Oxford: Oxford University Press, 1952.

WOOLDRIDGE, J. M. Econometric analysis of cross section and panel data. Cambridge: MIT Press, 2002.

WORLD HEALTH ORGANIZATION. WHO Expert committee on essential drugs. Geneva: World Health Organization, 1999.

Recebido em: 22/11/2010. Aceito em: 12/03/2014. 